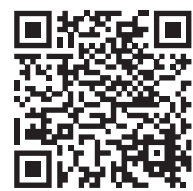
\footnotetext{
Debriefing, recomendaciones, a distancia, digital, capacitación docente.
}

Palabras clave:

Keywords: Debriefing, recommendations, distance, digital, teacher training.

\footnotetext{
* Cáncer Center Tec 100. Querétaro. México.

¥ SimMx. Querétaro.

México.

$\S$ Universidad

Autónoma de Chile.

ף Hospital de Simulación,

Universidad Andrés

Bello, Chile.

॥ Programa de

Anestesiología,

Universidad Andrés

Bello, Chile.
}

Recibido: 02/03/2021

Aceptado: 25/03/2021

doi: $10.35366 / 99864$

\section{Debriefing usando medios digitales: experiencia de un taller realizado en Latinoamérica y recomendaciones para su implementación}

\author{
Debriefing using digital media: experience of a workshop held \\ in Latin America and recommendations for its implementation
}

\author{
Juan Manuel Fraga-Sastrías, ,‡ Victoria Sotomayor,§ Raquel Espejo-González, \\ Eugenio Sanhueza-Herreros, "Mariana Alejandra Sierra-Murguía*
}

\section{RESUMEN}

Introducción: La pandemia de COVID-19 ha obligado a incorporar una serie de soluciones educativas, entre ellas el debriefing usando medios digitales (debriefing en línea). Con la finalidad de que docentes en Latinoamérica desarrollen competencia en este rubro se diseñó y realizó un curso de debriefing usando medios digitales del que se recogió información durante los ejercicios y en encuestas al final del curso y varias semanas después. Objetivos: Describir esta experiencia e identificar aspectos relevantes en la realización de un debriefing en línea. Material y métodos: Se realizaron cuatro cursos de 10 horas con 67 participantes en total entre agosto y diciembre de 2020. Se recogió información durante varios ejercicios en el curso y en encuestas de finalización y después de algunas semanas. Se analizó el contenido bajo la metodología Bottom-Up contando respuestas por unidades semánticas identificadas. Resultados: Se observó una serie de factores tecnológicos, metodológicos y otros asociados a la realización de debriefing en línea vs. debriefing presencial como la selección y conocimiento de plataformas, definición de reglas claras, respeto a la metodología, entre otros. De manera interesante se identificaron algunas ventajas como la flexibilidad, apego a la metodología, etcétera. Conclusiones: La experiencia del curso realizado fue útil para los participantes, y de la información recabada se puede tomar una serie de recomendaciones para quien desea incorporar esta técnica.

\section{ABSTRACT}

Introduction: The COVID-19 Pandemic has forced the introduction of a series of learning solutions among them online debriefing. With the aim of helping Latin-American healthcare teachers develop competency in doing it an online course was designed a provided online. During the course (on exercises) and after it by surveys at the end and several weeks later information was gathered. Objectives: Describe this experience and identify relevant aspects of the online-debriefing. Material and methods: Four courses were realized with a total of 67 participants between August and December 2020. Information was gathered during group exercises during the course, a survey at the end of the course and another survey sent several weeks after ending the course. We realized content analysis using Bottom-Up methodology counting answers by semantic units identified. Results: We found a number of technical, methodological and other aspects associated to the realization of online-debriefings vs. presential debriefings such as platform selection and knowledge, establishing clear rules, following the appropriate methodology among others. Interestingly some participants identified some advantages of online debriefing such as its flexibility, easier to follow methodology, etc. Conclusions: The experiences was useful for the participants and from the information gathered a number of recommendations can be used by people willing to incorporate online-debriefings.

\section{INTRODUCCIÓN}

$\Lambda$ partir de enero de 2020 la pandemia de Acovid-19 en el mundo, ha modificado la vida de todos en diversos aspectos: educativo, social, económico, en el otorgamiento de servicios de salud, entre muchos otros. En el caso de la educación de profesionales de la salud, ésta se ha tenido que ver modificada, por un lado, reduciendo o eliminando la presencialidad de los estudiantes
Citar como: Fraga-Sastrías JM, Sotomayor V, Espejo-González R, Sanhueza-Herreros E, Sierra-Murguía MA. Debriefing usando medios digitales: experiencia de un taller realizado en Latinoamérica y recomendaciones para su implementación. Rev Latinoam Simul Clin. 2021; 3 (1): 15-21. https://dx.doi.org/10.35366/99864 
en los espacios académicos y por otro, ajustando los contenidos de acuerdo a las nuevas necesidades emergentes producto de la pandemia. ${ }^{1}$

Una de las estrategias educativas que más se ha sacrificado durante esta pandemia es la simulación clínica y sus diversos componentes, entre ellos el debriefing. Estas necesarias adaptaciones pedagógicas han puesto de manifiesto el poder y el potencial de la educación virtual. Sin embargo, la facilitación de debriefings a través de plataformas virtuales plantea desafíos en muchos aspectos, especialmente en mayor esfuerzo y tiempo para garantizar la eficacia de las intervenciones educativas basadas en la simulación, directrices prácticas que apoyen un aprendizaje virtual significativo en la transición de los debriefings presenciales a los virtuales. ${ }^{2}$

Existen diversas experiencias y opiniones en distintos países del mundo ${ }^{2-5}$ acerca del debriefing realizado a distancia utilizando medios digitales como una medida para solucionar, en parte, las necesidades educativas en el área de la salud.

El curso constó de cuatro sesiones sincrónicas y actividades asincrónicas que debían ser realizadas por los participantes, las cuales se describen en la
Tabla 1. La tercera sesión se llevó a cabo en grupos pequeños con la finalidad de poder llevar a cabo una reflexión más profunda sobre la práctica de los debriefings que los participantes realizaban. Durante el curso se dividió a los participantes en grupos pequeños para recoger opiniones y reflexiones en torno a preguntas sobre la realización de debriefings y particularmente sobre los debriefings utilizando medios digitales. Dichas opiniones fueron recabadas empleando documentos compartidos (Google Drive) para posteriormente discutirlas y analizarlas.

El objetivo del presente artículo es describir la experiencia de un curso de entrenamiento para la realización de debriefing usando medios digitales (debriefing en línea) e identificar aspectos relevantes a considerar en su realización de acuerdo a los participantes del curso mediante un análisis de contenido.

\section{MATERIAL Y MÉTODOS}

Tipo de estudio: descriptivo, prospectivo.

Procedimiento: a partir de las experiencias internacionales ${ }^{2-5}$ se diseñó un curso de debriefing usando medios digitales con la finalidad de que los

Tabla 1: Programa del taller de debriefing usando medios digitales.

\begin{tabular}{|c|c|c|c|}
\hline Sesión/tema & Modalidad & Objetivo de la sesión & $\begin{array}{l}\text { Duración } \\
\text { (minutos) }\end{array}$ \\
\hline Introducción & $\begin{array}{l}\text { Sincrónica, en línea } \\
\text { Con docente }\end{array}$ & $\begin{array}{l}\text { Introducción de los participantes } \\
\text { Repaso de la técnica de debriefing } \\
\text { Identificar herramientas que pueden usarse para realizar debriefing en línea } \\
\text { Enumerar consideraciones durante cada etapa del debriefing al hacerlo en línea }\end{array}$ & 120 \\
\hline $\begin{array}{l}\text { Práctica } \\
\text { debriefing }\end{array}$ & $\begin{array}{l}\text { Asincrónica } \\
\text { Sin docente }\end{array}$ & $\begin{array}{l}\text { Realizar debriefing (clínico o educativo) y grabarlo, revisarlo y analizarlo para } \\
\text { discusión en próxima sesión en línea }\end{array}$ & 90 \\
\hline $\begin{array}{l}\text { Reflexión de la } \\
\text { experiencia }\end{array}$ & $\begin{array}{l}\text { Sincrónica, en línea } \\
\text { Con docente }\end{array}$ & $\begin{array}{l}\text { Revisar la experiencia de los ejercicios independientes } \\
\text { Profundización en estrategias tecnológicas para mejorar el debriefing: tipos de } \\
\text { plataformas, pros y contras de cada una } \\
\text { Desafíos del debriefing en línea }\end{array}$ & 120 \\
\hline $\begin{array}{l}\text { Práctica } \\
\text { debriefing } 2\end{array}$ & $\begin{array}{l}\text { Asincrónica, } \\
\text { sin docente }\end{array}$ & $\begin{array}{l}\text { Profundizar en el uso de herramientas digitales durante uno de sus debriefing. Grabarlo } \\
\text { y mostrar en la siguiente sesión para ejercicio en grupos pequeños }\end{array}$ & 90 \\
\hline $\begin{array}{l}\text { Revisiones en } \\
\text { grupos pequeños } \\
\text { de debriefings }\end{array}$ & $\begin{array}{l}\text { Sincrónica en línea } \\
\text { con el docente }\end{array}$ & Observación de vídeo y debriefing en grupos pequeños & 120 \\
\hline \multirow[t]{2}{*}{$\begin{array}{l}\text { Sesión final y } \\
\text { evaluación }\end{array}$} & $\begin{array}{l}\text { Sincrónica, en línea } \\
\text { con docente }\end{array}$ & $\begin{array}{l}\text { Reflexión final de la experiencia. Planes personales de incorporación a la práctica. } \\
\text { Ejercicio: recomendaciones para debriefing en línea. Evaluación del docente y el curso }\end{array}$ & 60 \\
\hline & & Total & 10 horas \\
\hline
\end{tabular}

En negritas aparecen los ejercicios que fueron analizados para fines de identificar recomendaciones para el debriefing digital. 
Tabla 2: Ejercicios durante el curso y opiniones recogidas.

\begin{tabular}{|c|c|}
\hline Ejercicio & Opiniones recogidas \\
\hline $\begin{array}{l}\text { Desafíos } \\
\text { del debriefing en línea }\end{array}$ & $\begin{array}{l}\text { Se plantearon situaciones hipotéticas y se solicitó a los participantes que propusieran } \\
\text { soluciones para prevenir/mitigar problemas durante el debriefing, antes y durante el mismo }\end{array}$ \\
\hline $\begin{array}{l}\text { Recomendaciones para } \\
\text { debriefing en línea }\end{array}$ & $\begin{array}{l}\text { Al final del curso se solicitó a los participantes que propusieran recomendaciones para } \\
\text { otras personas que desearan realizar un debriefing usando medios digitales }\end{array}$ \\
\hline
\end{tabular}

participantes de habla hispana pudieran utilizar esta técnica por medio de servicios de teleconferencia para enriquecer experiencias educativas u otras necesidades en que el debriefing presencial haya sido útil y que no haya sido posible implementar por las restricciones que representan las medidas sanitarias secundarias a la pandemia de COVID-19. Como fundamento teórico se utilizaron las experiencias antes mencionadas..$^{2-5}$ Aunque existen diversos modelos y métodos para realizar el debriefing ${ }^{2-6}$ para el curso en cuestión utilizamos la metodología propuesta por Steinwachs, ${ }^{7}$ que se usa ampliamente en diversos cursos de debriefing en el mundo, haciendo referencia a otras metodologías. ${ }^{6-9}$ El programa del curso se envió a las siguientes sociedades científicas: Federación Latinoamericana de Simulación Clínica, Sociedad Chilena de Simulación, Sociedad Argentina de Simulación Médica y Red Nacional de Educadores en Simulación Clínica (México) para su revisión y auspicio (aval).

Participantes: se incluyeron 67 participantes que tomaron alguno de los cuatro cursos impartidos entre agosto y diciembre de 2020 con una duración de tres semanas cada versión y con la agenda mostrada en la Tabla 1. De los 67 participantes, 26 (39\%) eran de Chile, 16 (24\%) de Perú, 15 (22\%) de Argentina, cuatro (6\%) de México, tres (4\%) de Colombia y un participante $(1.5 \%)$ de Brasil, Ecuador y Uruguay respectivamente. En cuanto al contexto en que se ha utilizado el debriefing por medios digitales 30 personas respondieron la encuesta correspondiente, ubicando a 21 (70\%) en educación de pregrado, cuatro (13.33\%) en educación de postgrado, 10 (33.33\%) en educación continua, dos (6.66\%) en actividades clínicas y uno (3.33\%) en investigación, siendo posible que una misma persona lo utilice en dos o más contextos. De acuerdo a la encuesta de salida utilizando el indicador Net Promoter Score (NPS) se tuvieron 66 promotores, uno pasivo y 0 detractores (NPS: 98.5). ${ }^{10}$

Instrumentos: durante la realización del curso se tomaron opiniones de los participantes en diversas ocasiones en torno a la experiencia de realizar debriefings en línea. En la Tabla 2 se resumen los ejercicios y las reflexiones documentadas y analizadas. Al final del curso se aplicó una encuesta de satisfacción y semanas después se envió a los participantes otra encuesta con el software Survey Monkey sobre la aplicación de estos conceptos en su práctica cotidiana, desafíos, ventajas y desventajas (Tabla 3).

Análisis de resultados: la información recogida en los ejercicios durante el curso y en la encuesta que se realizó semanas después de finalizado el curso, se estudió de manera independiente mediante un análisis de contenido del tipo BOTTOM-UP, ${ }^{11}$ es decir, del contenido a la teoría, generando categorías a partir de las respuestas de los participantes y contando la frecuencia de las unidades semánticas generadas.

\section{RESULTADOS}

Los objetivos del estudio fueron describir la experiencia de un curso de entrenamiento para realizar debriefing usando medios digitales y la identificación de aspectos relevantes a considerar en su realización.

En relación al objetivo de describir el curso de entrenamiento, utilizamos la información de la encuesta realizada unas semanas posteriores al curso. De los 67 participantes, obtuvimos respuestas de $30(44.8 \%)$ de ellos. De acuerdo a la pregunta sobre si su capacidad para realizar debriefing utilizando medios digitales había mejorado, 29 (96.67\%) indicaron que sí.

Los aspectos que consideraron relevantes de acuerdo a la encuesta posterior para hacer el debriefing en línea se mencionan en la Tabla 4, siendo más relevantes la planeación y anticipación (incluyendo proponer reglas y anticiparse a posibles situaciones problemáticas), además de revisar aspectos técnicos (selección adecuada de plataforma, revisar conexión, micrófonos, cámaras, etcétera). 
A su vez, los participantes reportaron algunas ventajas y desafíos de hacer el debriefing en línea (Tabla 5). Dentro de las principales ventajas reportadas la más frecuente fue la optimización de recursos, que las sesiones pueden ser grabadas y reanalizadas, además de ser más adaptables al contexto de los participantes. En cuanto a los desafíos reportados se identifican en mayor frecuencia problemas de conectividad, falta de conocimiento sobre la tecnología, mantener la participación activa, y el diseño de escenarios reales.

Los participantes también reportaron durante el curso aspectos a considerar para mejorar la experiencia del debriefing en línea. Éstos se describen en la Tabla 6. Dentro de los aspectos más relevantes se menciona nuevamente la adecuada selección de la plataforma, planeación, contar con estructura (mantener la estructura del debriefing), apego a la metodología con reglas de uso de cámaras (activadas) y audio silenciado (excepto cuando haya participación). Otro aspecto relevante reportado fue el uso de un co-debriefer.

Por último, se les pidió, también durante el curso, una lista de acciones que pudieran hacer para prevenir los posibles problemas antes, durante y después de la sesión. Dentro de lo más relevante, nuevamente fue la importancia de las reglas (incluyendo uso de cámaras, micrófonos, vestimenta, etcétera), el uso del chat de forma paralela y tener una introducción previa al curso (ya sea con un documento o vídeo previo).

En cuanto a los cambios en la manera de hacer debriefing en línea reportados en la encuesta posterior, el más frecuente fue mayor estructura/ orden, más uso de tecnología, optimización en uso de tiempo, mayor cumplimiento de objetivos, más autoeficacia para la realización de debriefing, y mayor percepción de beneficios (Tabla 7).

\section{DISCUSIÓN}

Cuando se habla de educación a distancia, es relevante considerar que ésta cumpla con estándares globales y colectivos, con una propuesta que tenga sentido/significancia para los participantes de diversas culturas digitales $(X)$. El debriefing a distancia incorpora todos los componentes del debriefing tradicional presencial, por lo que asegura el aprendizaje en un entorno protegido, abierto, flexible y motivante para los participantes. Sin embargo, se hace imprescindible el trabajo colaborativo y la revisión constante de procesos que permitan

\section{Tabla 4: Aspectos relevantes a considerar en el} momento de realizar un debriefing en línea.

Planeación/anticipación 15

Aspectos técnicos (conexión, plataformas, etcétera) 14

Procedimientos debriefing virtual 4

Seguridad de participantes 4

Encuadre

Experiencia del facilitador

Manejo de grupo

Participación

Realismo

Total

\section{Tabla 3: Encuesta posterior al curso.}

1. ¿Autoriza utilizar la información para fines de investigación? Sí No

2. ¿En qué ámbitos utiliza el debriefing usando medios digitales?
a. Educación de pregrado
b. Educación de postgrado
c. Educación continua
d. En actividades clínicas
e. Otro

3. Después de tomar el curso “Debriefing usando medios digitales” ¿considera que mejoró su capacidad para facilitar un debriefing en línea?
a. Sí
b. No

4. ¿Cuáles considera que son los aspectos más relevantes a tomar en cuenta durante un debriefing en línea?

5. ¿Qué ventajas ha observado al realizar un debriefing en línea vs. un debriefing presencial?

6. ¿Cuáles han sido los desafíos más importantes en la realización de un debriefing en línea?

7. ¿Cómo ha cambiado su manera de hacer un debriefing al hacerlo en línea? 
Tabla 5: Ventajas y desafíos del debriefing usando medios digitales.

¿Qué ventajas ha observado al realizar un debriefing en línea vs. uno presencial?

$\begin{array}{lr}\text { Optimización } & 7 \\ \text { Grabar } & 6 \\ \text { Reanalizar } & 4 \\ \text { Recursos } & 4 \\ \text { Adaptable a contexto } & 3 \\ \text { Ninguna } & 3 \\ \text { Tiempo } & 3 \\ \text { Distancia } & 2 \\ \text { Más apertura por interacción en línea } & 2 \\ \text { No sabe } & 2 \\ \text { Número de participantes } & 2 \\ \text { Costos } & 1 \\ \text { Dinámica } & 1 \\ \text { Herramientas digitales } & 1 \\ \text { Menos limitaciones } & 1 \\ \text { Pensamiento crítico } & 1 \\ \text { Personalización } & 1 \\ \text { Planificación } & 1 \\ \text { Seguridad } & 1 \\ \text { Total } & \mathbf{4 6}\end{array}$

\section{¿Cuáles han sido los desafíos más importantes en la realización de un debriefing en línea?}

$\begin{array}{lr}\text { Conectividad } & 7 \\ \text { Conocimiento de tecnología } & 6 \\ \text { Mantener participación } & 5 \\ \text { Escenarios } & 4 \\ \text { Comunicación } & 3 \\ \text { Mantener atención } & 3 \\ \text { Capacitación } & 2 \\ \text { Multitask } & 2 \\ \text { Validación de la herramienta } & 2 \\ \text { No sabe } & 1 \\ \text { Uso de cámara } & 1 \\ \text { Total } & 36\end{array}$

visualizar diversos aspectos como el manejo de recursos tecnológicos, el acceso y el tiempo que se debe dedicar a grupos virtuales para aclarar temas y gestionar el debate, lo que demuestra el esfuerzo adicional que se requiere para lograr la cohesión del grupo en los entornos virtuales. ${ }^{2}$

La crisis actual ofrece experiencias virtuales de cursos didácticos y clínicos que llevan al debriefing a la vanguardia del aprendizaje en la educación superior. ${ }^{3}$ Es importante compartir experiencias de debriefing virtual para intercambiar conocimientos, debatir y conectar ideas e identificar y aplicar nuevos conceptos y soluciones ${ }^{4}$ cuando no es posible hacerlo de manera presencial.

La Asociación Internacional de Enfermería para la Simulación Clínica y el Aprendizaje (INACSL) adicionalmente recomendada por la Society Simulation in Healthcare (SSH), sugiere que el debriefing puede llevar a nuevas interpretaciones por parte de los participantes; de hecho, la reorganización cognitiva es esencial para el aprendizaje. Las habilidades de la persona a cargo del debriefing son relevantes para asegurar los mejores resultados posibles en el aprendizaje ${ }^{5}$ en este sentido la propuesta a distancia incorpora la capacitación docente específica en debriefing a distancia, para potenciar el desempeño, y esta experiencia se ve enriquecida aún más cuando el facilitador ha tenido la oportunidad de generar debriefing presencial.

Las condiciones sanitarias actuales han obligado a las instituciones educativas a encontrar soluciones creativas para poder continuar con su labor educativa, y la posibilidad de utilizar el debriefing por medios digitales permite que al menos ese componente de la simulación clínica se realice sin riesgos para los participantes.

En esta aproximación a la técnica de debriefing usando medios digitales o debriefing en línea en Latinoamérica hemos identificado lo que podrían ser aspectos relevantes a considerar cuando se planea incorporar esta estrategia.

Tanto en los ejercicios efectuados por los participantes como en la encuesta aplicada unas semanas después, encontramos aspectos relevantes para la realización de un debriefing en línea, entre los que podemos mencionar:

\section{Aspectos técnicos}

Entre los aspectos técnicos más destacables se incluyen: conectividad, conocimiento de la tecnología, selección de la plataforma adecuada, seguridad informática (prevención de ingreso de participantes no deseados, por ejemplo), grabación de la sesión, uso de cámaras y micrófonos, niveles de audio, familiarización con la plataforma que se utilice, uso de chat y otras alternativas de comunicación con la finalidad de mejorar la comunicación, contar con alternativas 
de conexión (por ejemplo, el uso de celular si la computadora falla).

\section{Aspectos metodológicos y de planeación}

Quizá el aspecto metodológico de mayor importancia encontrado fue el de establecer reglas claras y comunicarlas anticipadamente (también llamadas reglas de "netiqueta"). Sin embargo, se mencionó con frecuencia la necesidad de respetar la metodología del debriefing, tener una buena planeación, planes alternativos (plan b), equipo de soporte disponible.

\section{Otros aspectos que considerar}

Otros aspectos considerados por los participantes son los de la necesidad de capacitarse en la rea-

Tabla 6: Ejercicio final. ¿Qué recomendaciones (tips) darías a quien realice un debriefing en línea?

\begin{tabular}{lc} 
Aspecto a considerar & $\mathrm{n}$ \\
\hline Seleccionar plataforma & 23 \\
Planeación & 18 \\
Audio silenciado & 12 \\
Cámara activada & 12 \\
Cuidar la metodología & 12 \\
Estructura & 12 \\
Tener equipo de soporte & 11 \\
Piloto previo & 9 \\
Reglas & 9 \\
Tener “plan b” & 9 \\
Revisar comunicación no verbal & 8 \\
Promover participación & 6 \\
Seguridad & 6 \\
Objetivos claros & 5 \\
Comunicación permanente & 4 \\
Consentimiento & 4 \\
Guía & 4 \\
Revisar conexión & 4 \\
Chat & 4 \\
Conocer público & 4 \\
Etiquetas & 3 \\
Cuidar entorno & 3 \\
Evitar juicios & 3 \\
Grabar & 3 \\
Coner monitor extendido & 2 \\
Efectos emocionales & 2 \\
Total & 2 \\
& 3 \\
\hline
\end{tabular}

Tabla 7: ¿Cómo ha cambiado su manera de realizar debriefing al hacerlo en línea?

$\begin{array}{lr}\text { Estructura/orden } & 6 \\ \text { Tecnología } & 6 \\ \text { Tiempo } & 4 \\ \text { Cumplimiento } & 3 \\ \text { Autoeficacia } & 2 \\ \text { Beneficios } & 2 \\ \text { No noto diferencias } & 2 \\ \text { No tengo experiencia } & 2 \\ \text { Variables externas } & 2 \\ \text { Eficiencia } & 1 \\ \text { Monitoreo } & 1 \\ \text { Total } & 31\end{array}$

lización de debriefing y debriefing en línea, con la ventaja de que poder ser grabado y analizado permite procesos de educación, supervisión o mejora continua. Mencionan como desafío el lograr mantener la atención de los participantes, y la necesidad de desarrollar estrategias para promover su participación, por ejemplo, dirigirse individualmente a los participantes.

Además, observamos que los participantes hallaron un número importante de ventajas en la realización del debriefing en línea, entre las que mencionaron: aspectos como mejor uso del tiempo, poder hacerlo a distancia, poder organizarse mejor, grabar y analizar posteriormente el debriefing, entre otros y un número limitado de desventajas, más asociadas al uso de tecnología como los problemas de conexión, comunicación, o estrategias para mantener la atención y participación de los participantes. Un punto que nos llamó la atención es que varios participantes mencionaron que hacer el debriefing en línea les permitió organizarse y estructurar mejor el debriefing, lo que fue un hallazgo sobresaliente que indica que esta metodología podría llegar a facilitar la incorporación del debriefing en algún segmento docente que no se siente tan cómodo realizándolo de manera presencial. El desafío tecnológico no es exclusivo del debriefing en línea y es seguramente uno de los aspectos a considerar al implementar un programa que incluya debriefing en línea. Esta necesidad de planeación no es algo nuevo en educación a distancia; ${ }^{12}$ sin embargo, las necesidades producto de la pandemia COVID-19 1 lo han vuelto a hacer patente.

A pesar de que este trabajo se limitó sólo a 67 participantes en cuatro cursos, y sólo a información cualitativa, consideramos que la 
experiencia puede servir como un referente en Latinoamérica que refuerza lo descrito por otros autores en distintas regiones geográficas y culturales. ${ }^{2-5}$ El formato de curso utilizado (en línea) aparentemente resultó útil a los participantes y lo recomendarían a otros colegas. En futuros estudios podría hacerse una evaluación de la competencia en la realización del debriefing en los participantes y no sólo tomar en cuenta su autopercepción.

\section{AGRADECIMIENTOS}

A todos los participantes que tuvieron la confianza de seguir estos cursos y responder a nuestras encuestas de manera abierta y honesta.

\section{REFERENCIAS}

1. Cayo-Rojas CF, Agramonte-Rosell RC. Desafíos de la educación virtual en odontología en tiempos de pandemia COVID-19. Rev Cubana Estomatol. 2020; 57: 3. Consultado en: http://scielo.sld. $\mathrm{cu} /$ scielo.php?script $=$ sci_arttext \&pid $=$ S003475072020000300017\&lng=es\&tlng=es.

2. Cheng A, Kolbe M, Grant V, Ellen S, Hales R, Symon $B$, et al. A practical guide to virtual debriefings: communities of inquiry perspective. Advances in Simulation. 2020; 5: 18. Available in: https://doi. org/10.1186/s41077-020-00141-1.

3. Gordon RM. Debriefing virtual simulation using an online conferencing platform: lessons learned. Clinical Simulations in Nursing. 2017; 13: 668674. Available in: http://dx.doi.org/10.1016/j. ecns.2017.08.003.

4. Bradley CS, Johnson BK, Dreifuerst KT. Debriefing: a place for enthusiastic teaching and learning at a distance. Clinical Simulation in Nursing. 2020; 49: 16-18.

5. International Nursing Association for Clinical Simulation and Learning (INACSL). Standards of best practice: simulation. [Accessed February 17, 2020] Available in: https://www.inacsl.org/inacsl-standardsof-best-practice-simulation/.

6. Oriot D, Alinier G. (Traducción de Espejo R, Sanhueza E, Arias E). Manual para el debriefing en Simulación Clínica. Universidad Andrés Bello/Ril Editores. 2020

7. Steinwachs B. How to facilitate a debriefing. Simulation \& Gaming. 1992; 23: 186-195. doi: 10.1177/1046878192232006.

8. Fanning RM, Gaba DM. The role of debriefing in simulation-based learning. Simulation in Healthcare. 2007; 2 (1): 1-11.

9. Flanagan B. Debriefing: theory and techniques. En: Riley RH. Manual of simulation in health care. Oxford. 2008, pp. 155-170.

10. Reichheld F, Markey R. The ultimate question 2.0: How net promoter companies thrive in a Costumer-Driven World. Harvard Business Review Press. 2011.

11. Smith J. Quantitative versus qualitative research: an attempt to clarify the issue. Educational Researcher. 1983; 12: 3. Available in: https://doi. org/10.3102/0013189X012003006.

12. Ruíz BC, Dávila AA. Propuesta de buenas prácticas de educación virtual en el entorno universitario. Revista de Educación a Distancia. 2016; 49 (12): 1-21. Consultada en: https://revistas.um.es/red/article/ view/257681/193881.

Correspondencia:

Dr. Juan Manuel Fraga-Sastrías

Palma Latania 150-B1. Palmares. 76127.

Querétaro. México.

Tel: 442 2581-157

E-mail: docfraga@docfraga.com 